\title{
Junke Fu: benevolence in heart, mission and duties in practice
}

Submitted Apr 18, 2018. Accepted for publication Apr 24, 2018.

doi: $10.21037 /$ jtd.2018.05.07

View this article at: http://dx.doi.org/10.21037/jtd.2018.05.07

\section{Foreword}

"Why did you choose to become a doctor?"

"I was born and grew up in the countryside. In the 1970s, the medical conditions in the countryside were very dire, and the pain that diseases inflicted on the local people touched me deeply. At that time, I regarded being a medical doctor as a very sacred calling, since doctors save lives. Maybe I was destined to become a doctor. Looking back, I am more certain that I am so fortunate to be working in the medical field. Despite the ups and downs along the way over the years, I have always believed that as long as I take this career seriously and earnestly, love this profession, maintain my original motivation, continue to try and have perseverance, I will be a good doctor and will be successful."

\section{Expert's introduction}

Junke Fu, male, Professor, Chief physician and Director of the Department of Thoracic Surgery, The First Affiliated Hospital of Xi'an Jiaotong University. Dr. Fu is an expert on surgical thoracic treatment with minimally invasive surgery, lung transplantation and the diagnosis and surgical treatment of difficult and critical thoracic diseases. He is among those that have pioneered the minimally invasive surgery on esophageal cancer and robot-assisted minimally invasive surgery in China, especially in China's northwest region. He has been quite influential and has successfully trained doctors in dozens of hospitals on minimally invasive esophageal surgeries. Currently, he focuses on the accelerated recovery of esophageal cancer patients and has conducted a large number of basic science and clinical studies on preoperative preparation, and intraoperative and postoperative management of patients. By strengthening preoperative education, improving preoperative nutritional status, strengthening anesthesia management, optimizing surgical procedures and employing various effective postoperative management measures, he and his team have significantly accelerated the postoperative recovery of patients with esophageal cancer and improved their quality of life. Dr. Fu has participated in four national- level research projects such as the National Natural Science Foundation project, presided over two provincial research projects and published four books and more than 20 papers. The "Minimally invasive radical surgery on esophageal cancer" and "Clinical application of DCD donor lung transplantation" projects both won first prize of the New Clinical Treatments and New Technologies of Xi'an Jiaotong University in 2016. He is a committee member of the Cardiopulmonary Transplantation Group of the Organ Transplantation Sub-division of Chinese Medical Association; a member of the Standing Committee of Thoracic Surgery of the China Research Hospital Association; a member of the Standing Committee of the Specialty of Thoracic Surgery of the Cross-Strait Medical and Health Exchange Association; a member of the Thoracic Surgery Branch of the Chinese Medical Doctor Association; a member of Lung Cancer Collaboration Group of the Chinese Thoracic Surgery; a young member of the Greater China Thoracoscope Development and Promotion Committee; a member of the Minimally Invasive Surgery Specialty Committee of the Thoracic Surgery Branch of the Chinese Medical Doctor Association; a member of the Esophageal Specialty Committee of the Thoracic Surgery Branch of the Chinese Medical Doctor Association; the deputy director of the Thoracic Surgery Branch of the Shaanxi Medical Association; the alternate director of the Esophageal Cancer Specialty Committee of Shaanxi Province Anti-cancer Association; a member of the Standing Committee of the Lung Cancer Specialty Committee of the Shanxi Province Anti-cancer Association; a member of the Standing Committee of the Western Region Lung Cancer Collaborating Center; the deputy director of the Thoracic and Cardiovascular Surgery Department of the Xi'an Medical Association; and executive editor of the "Chinese Fournal of Lung Cancer". Dr. Fu has won the "Fifth Chinese Physician Award" and the "Chinese Humanities and Medicine Honorary Award" and has been recognized as the "Outstanding Individual for Earthquake Relief of the China Association for Science and Technology”. 


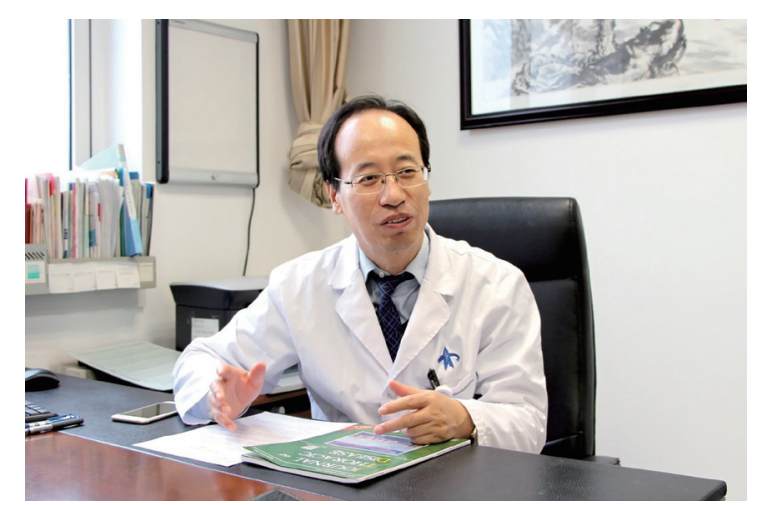

Figure 1 Professor Junke Fu. Professor Junke Fu was being interviewed by the editor from AME Publishing Company.

\section{Being a doctor is the best career}

In September 1981, aspiring to study the medical sciences, Junke Fu (Figure 1) entered the Department of Clinical Medicine at the former Xi'an Medical College, embarking on a journey into the medical field. In 2001, he began attending the graduate school at Sichuan University to pursue a $\mathrm{PhD}$ in Thoracic Surgery in West China Hospital. Since graduating in 2004, he has served as the director of the Department of Thoracic Surgery of the First Affiliated Hospital of Medical School at Xi'an Jiaotong University.

Each day, he rises at 6:20 am and arrives at the office at 7:20 am. He begins his shift at 7:30 am, makes his rounds at 8:00 am, and begins performing surgery at 8:30 am. Day in and day out, this cycle has repeated for decades. "How would you describe your current working status?" "I would say it's a 5+2," Dr. Fu answered with a grin. Three to six surgeries per day if it is an operation day, intramural MDT case discussions, extramural medical consultations and academic meetings, a surgical teaching position at the medical school, mentoring master and $\mathrm{PhD}$ graduate students, and often attending academic conferences on the weekend-these activities occupy Dr. Fu's daily life. For him, nearly every day is a workday.

The phrase "time is life" is literally true for doctors. Faced with such a high work intensity, Dr. Fu always feels that time is too short, yet he never retreats from his position on the clinical work, with unfettered enthusiasm for being a medical doctor. "I have been a doctor for 31 years, and the work of doctors can really solve some patients' problems; the more I work as a doctor, the more I feel that the job fits me. I love this job."

\section{Perform everything wholeheartedly and perform it from the patient's point of view}

China has the highest incidence of esophageal cancer in the world, accounting for more than $50 \%$ of the total cases, and the occurrence of esophageal cancer in China has a significant regional bias, as a poor area in northwest China is a high-risk region. Having grown up in the countryside of that region, Junke Fu witnessed the immense suffering and burdens the sickness had brought on the patients and their families in the poverty-stricken area, "I grew up in the countryside and saw the pain that my family members and many people around me in very poor economic conditions suffered. So as a doctor, I must do something for them."

In 2007, the concept of enhanced recovery after surgery (ERAS) was introduced in China. ERAS emphasizes a series of evidence-based optimized perioperative management measures that reduce physical and psychological traumatic stresses in surgical patients to achieve rapid recovery, representing a new concept and a novel therapeutic rehabilitation model in the 21 st century. Junke Fu said he first heard about the ERAS concept at a conference, "After the meeting, I was eager to know more about the idea of ERAS, which is a new concept in the management of patients during the perioperative period. The economic situation of patients with esophageal cancer in the northwestern region of China is often dire. I think if we apply this theoretical system to the clinical practice of esophageal cancer treatment and optimize and streamline all its clinical aspects, the patients will greatly benefit in various aspects, such as a reduction in the incidence of postoperative complications, thus reducing patients' suffering, accelerating patients' functional recovery, shortening their hospital stay and enabling them to return to society as soon as possible, decreasing the patients' hospitalization costs and, therefore, their economic burdens. This is a very worthy cause."

Only by employing ideas in practice can we turn ideas into reality. In recent years, Junke Fu has led a team to perform investigations of fast-track surgery on esophageal cancer, and the team has conducted a large number of basic and clinical studies on preoperative preparation, intraoperative treatment and postoperative management of the illness. Thus, how can ERAS be better applied to clinical practice? "We have applied the ERAS concept to every aspect of the perioperative period of esophageal cancer treatment, and a dedicated team is established to lead each stage. The teams are interdependent, which greatly facilitates the implementation of the ERAS concept 
in clinical practice. Through active collaboration among various departments, such as anesthesiology, respiratory, nutrition, cardiology and traditional Chinese medicine, we have developed a complete set of perioperative ERAS procedures for esophageal cancer treatment.”

Patients' understanding and true cooperation in the treatment process are prerequisites for the effective implementation of ERAS. Junke Fu stresses the need to have patience with the patients and communicate with them. "We should let the patients fully prepare themselves psychologically; for example, we should tell the patient that after the surgery, he or she may need a gastric tube, a feeding tube, or a urinating catheter. Otherwise, after the surgery, the patient can become very upset at the sight of these tubes."

The surgical procedure itself is crucial in a patient's rapid postoperative recovery. Junke Fu took the lead in performing minimally invasive surgery on esophageal cancer in the northwest region. Today, $85 \%$ of the surgeries are performed with thoracoscope and/or laparoscope in Dr. Fu's department. In 2016, the "Minimally invasive esophagectomy" by Dr. Fu's team won the first prize of New Clinical Treatments and New Technologies issued by Xi'an Jiaotong University. Regarding minimally invasive surgery, Dr. Fu notes that "minimal invasiveness" does not simply refer to the surgery in an anatomical sense; more importantly, it concerns functionality, so intraoperatively, we must pay special attention to protect important nerves, tissues and organs. When performing digestive tract reconstruction, we must choose the anastomotic method with which we are most familiar; it is understandable for young doctors to pursue new skills, but we cannot use patients as guinea pigs.

Compared with that of patients with lung cancer, the ERAS of patients with esophageal cancer is significantly more complicated. The majority of patients with esophageal cancer have malnutrition issues prior to surgery. The doctors in the enteral nutrition team would perform intensive nutritional measures based on the patient's condition prior to surgery to facilitate the patient's rapid postoperative recovery. Therefore, are there postoperative recovery programs for gastrointestinal functions that are both conducive to the patient's recovery and economy? By teaming with the traditional Chinese medicine team, Dr. Fu's team accelerates the recovery of gastrointestinal functions through adjuvant therapies using traditional Chinese medicinal herbs, enemas or nasal feeding and has achieved outstanding results. "We have also considered some methods that are both economical and effective to get patients to recover as quickly as possible, which is in line with the idea of ERAS. In the process of performing a gastric conduit, the stomach will inevitably be injured; however, the wounds will heal faster when we use traditional Chinese medicine, which leads to very good outcomes. If early postoperative interventions are performed, the gastric fluid of the patient will gradually become clear on the second or third day after surgery, so I think it is very worthwhile for us to explore traditional Chinese medicine in this regard."

Dr. Fu is thrilled by the robust development of minimally invasive surgery and the achievements of ERAS in the field of thoracic surgery in recent years. "The development of minimally invasive techniques in China is particularly rapid, and coupled with the clinical application of the concept of ERAS, it has significantly reduced the postoperative complications of thoracic surgery and shortened the average length of a hospital stay. These benefits are happening at every medical center." Perfecting his skill while increasing care of his patients, Dr. Fu has never paused his exploration of new surgical procedures that are more minimally invasive and more conducive to a patient's rapid recovery. On March 11, 2016, Dr. Fu performed two lung surgeries with the assistance of the da Vinci robot system. One was a very complex lung cancer resection of lesions with pleural adhesions and lymph node calcification, which represented the first da Vinci robot-assisted lung cancer resection in Shaanxi Province and paved the way for robot-assisted minimally invasive thoracic surgery in the province. On the morning of March 31, 2016, Dr. Fu successfully performed a robot-assisted esophageal hiatus hernia repair on an elderly male patient, which was also the first case in the province; the entire operation resulted in only $20 \mathrm{~mL}$ of blood loss, and on the second day after surgery, the patient was able to walk unassisted. To date, the Junke Fu team has performed nearly 100 robot-assisted surgeries.

Lung transplantation is a major surgical operation; if the application of ERAS in the field of thoracic surgery benefits patients, then applying the concept of ERAS in the perioperative management of lung transplantation is also of great significance. On November 23, 2006, the Department of Thoracic Surgery, led by Junke Fu, successfully performed their first allogeneic lung transplantation, and in 2015, they conducted the first DCD lung transplantation in Shaanxi Province. To date, seven patients have successfully received lung transplantation. Guided by the concept of ERAS, in the case of an uncomplicated lung transplant 
operation, the Junke Fu team encourages patients to get out of bed on the first or second day after surgery. With adequate preoperative preparations and appropriate postoperative management measures, lung transplant patients can be removed from the ventilator very soon after surgery and can quickly recover lung function. "The perioperative recovery of patients who have undergone lung transplantation has gone far beyond our imagination."

Facing these achievements, Junke Fu said, "As a doctor, as long as I have done everything wholeheartedly, I am always very pleased and happy when reflecting on these endeavors."

\section{Sometimes to cure, often to help, but always to comfort}

"Sometimes to cure, often to help, but always to comfort." Junke $\mathrm{Fu}$ consistently practices this ideal. "Whenever a patient approaches us seeking help, we would definitely do everything we can to help solving his or her problem."

On November 28, 2004, a serious coal mine disaster occurred in the Chenjiashan Coal Mine in Shaanxi Province, and Junke Fu immediately participated in a medical rescue team that went to Tongchuan, the disaster site. At 14:28 on May 12, 2008, a magnitude eight earthquake struck Wenchuan, Sichuan Province. After learning of the devastation caused by the earthquake, Junke $\mathrm{Fu}$ volunteered to serve in an earthquake medical relief team and was actively involved in rescue missions in the disaster area.

Junke Fu believes that these experiences are the same as those of everyday treatment on each patient, "As a doctor, my mission is to save lives and help the wounded. The longer I am a doctor, the more I realize that doctors should appreciate the sufferings of the unfortunate and ease their pains. In these dire circumstances, this feeling is even more intense; being a doctor, I have to stand up."

Junke Fu was once awarded with the "Humanities and Medicine Honorary Award" by the Ethics Committee of the Chinese Medical Association and the Fifth Chinese Physician Award. Discussing the patients who have deeply touched him, Junke Fu said: "People often say that patients are moved by doctors, but many a time I am also moved by my patients." Over a decade ago, Junke Fu performed lung cancer surgery on a patient who was a retiree of a construction company and is now over 80 years old. At eight o'clock each Chinese New Year, this old man calls Fu to give him his first New Year's greeting. "I am really touched. What I did was simply listen to bim when he was hospitalized, and I tried my best to relieve his pain from the illness, which is my job, but he bas been grateful to me for the rest of his life. As a doctor, I am very proud and glad to be held in such high regard by my patient."

With regard to the current tension between doctors and patients, Junke Fu discusses his own personal experience: " $A s$ a doctor, we must treat patients with a sincere heart and consider various problems really from the patients' perspective. Not only must we make our utmost effort to cure various diseases, but we must also deliver humane care to patients so that they can be strong when facing and overcoming difficulties."

In Junke Fu's opinion, the largest responsibility of a doctor is to continue to learn and improve his or her professional capabilities. "Only with superb medical skills are we able to relieve the pain of patients with complex and serious disease; this is most important."

With respect to a young doctor's career development, Junke Fu's recommendation is to "work hard, be a good person, engage yourself wholeheartedly in your work, love your work and love your patients."

\section{Interview}

FTD: From your experience, can you tell us bow to reduce the incidence of postoperative complications in minimally invasive thoracoscopic surgery for esophageal cancer?

Junke Fu: The incidence of esophageal cancer in China is the highest in the world; in recent years, minimally invasive thoracoscopic surgery on esophageal cancer has developed very rapidly in China. As a matter of fact, minimally invasive surgery is also within the concept of ERAS. Based on my personal experience, we must focus on certain problems during the operation.

"Minimal invasiveness" is not just "minimal invasiveness" in the anatomical sense that minimally invasive surgeries are merely those performed through a few very small incisions. We should focus on "minimal invasiveness" in the sense of functionality, i.e., reducing the stress on the body and preventing the functional decline of various organs; this represents "minimal invasiveness" at a higher level. Functional "minimal invasiveness" embodies the concept of ERAS, which is reflected in every aspect of the surgery. For example, we must be very careful to protect important nerves, such as the vagus and recurrent laryngeal nerves, and their functions while completely removing the tumor tissue in surgery; otherwise, the damage to the nerves will affect the postoperative recovery of the patient. Moreover, we also need to maximally protect organs around the esophagus, such as the trachea and tracheal membrane, because damage 
to these organs can lead to postoperative complications. The majority of minimally invasive surgeries on esophageal cancer patients require replacing the esophagus with the stomach. Thus, postoperatively, the stomach has the dual function of stomach and esophagus; therefore, it must be protected during surgery by avoiding excessive pulling or inadvertent damage during the peritoneal dissection. When constructing a gastric tube, we must focus on details such as blood supply to the stomach since good blood supply is an important condition for postoperative healing. In the case of a poor blood supply, the patient's postoperative healing will be very poor, leading to a high chance of anastomotic leakage.

However, when performing digestive tract reconstruction, we should consider the patient's specific conditions and choose the anastomotic technique with which we are most familiar. Anastomotic complications can seriously affect patients' quality of life, prolong their average length of hospital stay, increase the costs of hospitalization and bring additional suffering to the patients. Many young doctors are enthusiastic in pursuing novel surgical methods and are always attempting new methods and learning, but my recommendation is that they should use their most mature skills on patients, reducing the risk of anastomotic complications.

\section{FTD: You have organized multiple workshops on minimally invasive surgery on esophageal cancer. What do you think is the significance of conducting a workshop?}

Junke Fu: The economic development in China's northwestern region is relatively backward, and the level of medical technology is also lagging. In recent years, we have held a series of conferences, minimally invasive surgery and airway management workshops, and along with the MDT team, we have successfully trained some doctors from primary hospitals to perform endoscopic esophageal surgeries. We, in the northwestern region, are among the first to acquire the concepts of minimal invasiveness and ERAS, and having placed these ideas into clinical practice and accumulated certain experience, we feel that it is our responsibility and obligation to introduce and promote these new technologies and ideas to other medical personnel, thereby benefiting more patients. The goal of these conferences and workshops is to help students implement new ideas into clinical practice. We are very pleased to observe that through our efforts over the years, the primary hospitals have made great progress in the field of minimally invasive surgery.

\section{fTD: Compared with thoracoscopic surgery, what are the advantages of robot-assisted minimally invasive surgery? Can you talk about your experience? How do you see the future of robot-assisted surgeries?}

Junke Fu: We have performed nearly 100 robot-assisted surgeries that involve lung lobectomy, segmentectomy, sleeve lobectomy, esophagectomy and esophageal hernia repair. The advantages of robot-assisted surgery include the highly magnified three-dimensional field of view, which is very important for accurate surgical operation, and the mechanical arm operation, which effectively avoids the surgeon's slight movements during an operation and is more dexterous while easing the surgeon's fatigue. Robots will be more intelligent and smaller in size in the future, and even remote-controllable, which will make remote surgery a reality.

\section{FTD: Please briefly introduce the ERAS perioperative procedure for esophageal cancer patients in your hospital based on your experience.}

Junke Fu: Preoperative measures include preoperative education, preoperative airway management and improving the patient's preoperative nutritional status. The patient's understanding and true cooperation in the treatment process is the premise for implementing ERAS. During preoperative education, the nursing team explains the preand post-operative precautions to the patient. Perioperative airway management is an important component of ERAS, which occurs throughout the entire perioperative period. The airway management team evaluates the patient's lung function prior to surgery. If the patient's lung function and airway status are poor, necessary preoperative airway management measures will be taken, and airway training as well as intensive nebulized inhalation treatment will be performed based on the patient's specific conditions. Compared with the ERAS of patients with lung cancer, the ERAS of patients with esophageal cancer is more complicated; the majority of esophageal cancer patients have malnutrition problems prior to surgery. Doctors in the enteral nutrition group will evaluate the patient's nutritional status prior surgery and perform corresponding improvement measures based on the patient's condition.

In terms of intraoperative anesthetic management, 
we work closely with the anesthesiology department. Anesthesiologists will carefully examine the patient prior to surgery to provide appropriate anesthetic management measures during surgery, including intraoperative fluid and hypothermia management, to prevent metabolic disorders and other issues, which greatly shortens the recovery time after anesthesia as well as the indwelling time of tracheal intubation. When choosing the anesthesia method, except in the case of complex lung surgeries in which doublelumen endotracheal intubation is performed, singlelumen endotracheal intubation is adopted in the majority of esophageal surgeries to reduce damage to the airway during anesthesia. In recent years, in collaboration with the department of anesthesia, we have significantly shortened the postoperative intubation period and improved the outcomes of postoperative pulmonary complications. In 2015, we began performing tubeless (no intubation) chest surgeries, such as pulmonary bullae resection, lung nodule excisional biopsy, bilateral sympathectomy and mediastinal tumor resection, without endotracheal intubation, so patients receive more minimally invasive treatments and recover more quickly.

To prevent postoperative airway complications, we also employ effective measures such as intensive nebulized inhalation drug delivery and anti-infection treatment as early as possible. In recent years, through perioperative airway management, the number of patients who require postoperative sputum aspiration has significantly decreased. Except for some patients with poor health, rare patients had major airway complications. To prevent postoperative deep vein thrombosis, we examine the patient prior to surgery and perform Doppler detection on the veins of the lower extremities for a high-risk patient. After surgery, on special patient populations such as those with obesity, diabetes or hypertension, anticoagulation is performed as early as possible. Moreover, under the ERAS concept, we encourage patients to get out of bed as soon as possible.

In terms of postoperative pain control, we have adopted a combination analgesia method. In recent years, we have developed a scoring system for patients' postoperative pain experience. We use this system as a basis to determine whether intensive analgesic measures are needed for a specific patient. In terms of intubation management, whether it is lung or esophageal surgery, only a single chest drainage tube is postoperatively placed, and the diameter of the drainage tube is gradually reduced so the patient's pain can be alleviated. Regarding the management of urinary catheters, in some surgeries, such as pneumothorax, palmar hyperhidrosis or lung biopsy, in which the operation time is preoperatively estimated to be within one and a half or two hours, it is unnecessary to indwell a catheter. The smooth implementation of ERAS is inseparable from the efficient cooperation between the surgical and nursing teams, and our nursing team has also developed a series of procedures in urinary catheter management and will remove the catheter as soon as possible based on the patient's body fluid conditions.

\section{FTD: In what aspects of perioperative airway management do you think room for improvement remains?}

Junke Fu: In recent years, with the team efforts, the concept of perioperative airway management has been promoted; however, in practice, some problems remained. First, we have not intervened prior to the problem arising. For example, in the case of respiratory complications, measures such as intensive nebulized inhalation drug delivery and anti-infection therapy are often taken after a patient has already developed complications, which is less efficient. Second, currently in most hospitals, individualized and precise treatment plans are lacking, which should be corrected. For example, in the future, we would develop different treatment plans for patients with different conditions, such as chronic obstructive pulmonary disease (COPD), asthma and a smoking history. Third, although some clinical phenomena have caused widespread concern among doctors, their causes remain unknown and should be intensively investigated so that corresponding coping measures can be taken. For example, many doctors have noticed that the incidence of long-term postoperative cough is significantly higher in female patients than in male patients; the cause is unclear. Regarding preoperative airway management in female patients, we have to conduct additional investigations to determine which additional measures should be taken.

\section{FTD: What is the significance of perioperative airway management for thoracic surgery?}

Junke Fu: All operations in thoracic surgery (lung, esophagus and mediastinum) have close relationship with the pleural cavity, and once the integrity of the pleural cavity is lost, the respiratory function of the lungs will be affected. However, in addition to some thoracic surgeries that can be performed without intubation, the majority of operations have to be performed with tracheal intubation, which is a physical intervention that, to a certain extent, 
causes damage to the airway. Therefore, thoracic surgery itself inherently determines the relatively high incidence of postoperative respiratory complications. Overall, relative to those of abdominal or gynecological and obstetric surgeries, perioperative airway management in ERAS of thoracic surgery is particularly important.

\section{TD: What experience do you want to share with us regarding the ERAS of esophageal cancer?}

Junke Fu: Compared with lung cancer surgery, esophageal cancer surgery requires not only the complete removal of the tumor but also the reconstruction of the digestive tract, so postoperatively, patients will experience a long period of functional recovery to regain various functions such as swallowing, gastric emptying and intestinal functions. In recent years, we have accumulated some experiences in this regard in the treatment of esophageal cancer. Because each person's intestinal function is different, we have to postoperatively assess whether it is appropriate for a patient to begin eating based on the patient's appetite and intestinal function recovery status in combination with the patient's general condition; not all patients need to begin eating the third or fifth day after surgery. The timing must be individualized.

\section{FTD: Which direction do you think ERAS will develop in the future?}

Junke Fu: Prior to the idea of ERAS being developed, doctors were passive when facing the majority of the problems occurring to patients; whenever a patient had a problem, doctors attempted to find an answer. Having acquired the concept of ERAS, doctors are prone to take initiative to intervene in every pre-, intra- and postoperative aspect and to employ all possible measures in order to enable patients to recover quickly. If doctors can anticipate the patient's problems before they occur, employ effective measures to prevent or avoid the problems before they become emergences and begin the ERAS measures as early as possible, the highest level of ERAS will be achieved, providing profound benefits to the patients.

\section{FTD: What are your research priorities and directions for ERAS in the future?}

Junke Fu: In the future, China will face the aging problem, and the incidence of lung cancer is increasing annually. With regard to the accelerated recovery of lung cancer patients, we should gain a better understanding of how to achieve more precise and more individualized pulmonary rehabilitation for various patient populations, such as the elderly, patients with COPD and patients with diabetes; we will address these problems in the future. However, esophageal cancer is a disease with "Chinese Characteristics", and among the annual 450,000 new esophageal cancer cases in the world, approximately 250,000 emerge in China, where esophageal cancer is highly prevalent in economically underdeveloped regions such as mountainous and rural areas. The accelerated recovery of esophageal cancer patients enables them to recover quickly, saves hospitalization costs, and thus relieves the patient's economic burden. In this regard, we have already performed some exploratory work, such as integrative medicine and individualized treatment, but many problems remain, and we would solve these issues.

\section{Acknowledgements}

We wish to express our gratitude to Lili Liao and Siying Yan of the AME Publishing Company for their strong support and assistance for this article and to Liang $\mathrm{Li}$ of the AME Publishing Company for his support and assistance with the interview.

\section{Footnote}

Conflicts of Interest: The author has no conflicts of interest to declare.

(Science Editor: Jiaqi Li, JTD, jtd@amepc.org)
Cite this article as: $\mathrm{Li} \mathrm{J}$. Junke Fu: benevolence in heart, mission and duties in practice. J Thorac Dis 2018;10(Suppl 11):S1267-S1273. doi: 10.21037/jtd.2018.05.07 